NASA/TM-2001-211207

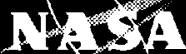

Experimental Results of Schlicher's Thrusting Antenna

Gustave C. Fralick

Glenn Research Center, Cleveland, Ohio

Janis M. Niedra

QSS Group, Inc., Brook Park, Ohio

\title{
Prepared for the
}

37th Joint Propulsion Conference and Exhibit cosponsored by the AIAA, SAE, AIChE, and ASME

Salt Lake City, Utah, July 8-11, 2001

National Aeronautics and

Space Administration

Glenn Research Center 


\section{Acknowledgments}

The authors would like to thank Carl Lorenzo and Ken Weiland, who constructed the antenna, John Wrbanek, who helped with the figures, and Dr. Norm Wenger, who contributed to the analysis.

Available from

NASA Center for Aerospace Information

7121 Standard Drive

National Technical Information Service

Hanover, MD 21076 


\section{EXPERIMENTAL RESULTS OF SCHLICHER'S THRUSTING ANTENNA}

\author{
Gustave C. Fralick \\ National Aeronautics and Space Administration \\ Glenn Research Center \\ Cleveland, Ohio 44135
}

\author{
Janis M. Niedra \\ QSS Group, Inc. \\ Brook Park, Ohio 44142
}

\begin{abstract}
Experiments were conducted to test the claims by Rex L. Schlicher et.al. (Patent 5,142,861) that a certain antenna geometry produces thrust greatly exceeding radiation reaction, when driven by repetitive, fast rise and relatively slower decay current pulses. In order to test this hypothesis, the antenna was suspended by strings as a $3 \mathrm{~m}$ pendulum. Current pulses were fed to the antenna along the suspension path by a very flexible coaxial line constructed from loudspeaker cable and copper braid sheath. When driving the antenna via this cabling, our pulser was capable of sustaining $1200 \mathrm{~A}$ pulses at a rate of 30 per second up to a minute. In this way, bursts of pulses could be delivered in synch with the pendulum period in order to build up any motion. However, when using a laser beam passing through a lens attached to the antenna to amplify linear displacement by a factor of at least 25 , no correlated motion of the beam spot could be detected on a distant wall. We conclude, in agreement with the momentum theorem of classical electromagnetic theory, that any thrust produced is far below practically useful levels. Hence within classical electrodynamics, there is little hope of detecting any low level motion that cannot be explained by interactions with surrounding structural steel and the Earth's magnetic field.
\end{abstract}

\section{Introduction}

This paper describes the attempt to measure the thrust of the "Non-Linear Electromagnetic Propulsion System" (NEMPS), patent no. 5,142,861, (Ref. 1) and described at the $31^{\text {st }}$ Joint Propulsion Conference (Refs. 2-5). The device supposedly generates a mechanical force when electric current is sent through it. Such a device, if it produced more than the miniscule thrust of a photon rocket, would be a boon for deep space, perhaps, even interstellar, missions. No longer would it be necessary to carry half the mass of the mission in fuel. Just connect your electric thruster to a power source, such as a radioisotope heat source, and off you are to the nearest star!
After an attempt to acquire a thruster from the developer, we decided to build our own, using the patent drawings and other published material (Refs. $1-5$ ) as construction guidelines. We also built our own pulse power supply, again relying on the sketchy descriptions provided in the references. Since we received no direct help from the developer, the device is not a perfect reproduction, but likely works as well. The reasons for this statement are given at the end of this paper.

\section{Principle of Operation of the NEMPS thruster}

According to the device description given in Ref. 2, "the rigid three dimensional geometric asymmetries in the loop antenna structure cause a magnetic field density gradient internal to the antenna structure along a single axis. This magnetic field density gradient then causes an imbalance in the internal magneticmechanical forces that normally result from the interactions of the loop antenna's internal magnetic field with the current in the conductors of the loop antenna's structure, as described by the Lorentz force law." The thruster is powered by a pulsed power supply "designed to provide the proper waveform to the antenna structure at an impedance matching the load impedance of the antenna. The rise time and shape of the input current waveform is crucial to maximizing the production of reaction thrust." However, neither the pulse parameters nor the reason for their criticality are given.

\section{Description of Experiment}

Conducting the experimental test of the claims of the NEMPS thruster turned out to present a different set of problems than was expected. Initially we thought that we would see evidence of some force being produced, and then have to eliminate any spurious or non-relevant forces, such as interaction with the earth's magnetic field, or with the structural steel of the building, or perhaps even the electrostatic precipitator effect. But we really observed no evidence of any force 
whatsoever. Thus we were reduced to setting an upper limit for the force produced by the thruster.

The first step in testing the NEMPS thruster was to build it. As can be seen from the drawing, (Fig. 1), and our implementation of it (Fig. 2) the device is a coaxial transmission line, the outer conductor of which blooms into a petal like arrangement. Four bundles of two conductors each lead from the petals back to the center conductor to complete the circuit. In our embodiment of the NEMPS thruster, the center conductor is a piece of copper rod. $1 / 4$ " in diameter and 30 " long. The portion of the outer conductor between the input and the petals is a piece of copper pipe, $1 \frac{1}{2}$ " in diameter. The distance from the petals to the input is $5 \frac{1}{2} "$. The petals, there are eight of them, are made of $1 / 8$ " aluminum plate. They fan out at an angle of approximately 45 degrees to a diameter of 6", and then are parallel to the central axis for another $2 \%$ ". The four conductor bundles run parallel to the axis for 10", then back to the center conductor at an angle of 50 degrees. The mass of the thruster is approximately $1.8 \mathrm{~kg}$.

The second step in testing the thruster was to provide power to it. The power is fed through a pair of 14 gauge wires. surrounded with copper braid, effectively making a high current coaxial cable.

The shape of a typical current pulse when operating into a $0.05 \Omega$ resistive load is shown in Fig. 3. Here the pulse height is $2400 \mathrm{~A}$, with rise and fall times of about $13 \mu$ s and $314 \mu$ s, respectively. However, when driving the thruster via our made-up coaxial cables, the amplitude was reduced by the increased impedance to about 1200 amps peak, and the rise and fall times increased to about $20 \mu \mathrm{s}$ and $400 \mu \mathrm{s}$, respectively. The pulse repetition rate was 30 pulses per second. To the best of our ability, we duplicated the pulse shape shown in Fig. 4, which is a sketch from Ref. 1.

This brings us to actually measuring the force. After considering various schemes, we decided to hang the thruster as a pendulum. This provides a low friction mount, and a convenient means for measuring the force. Any force produced by the thruster produces a displacement from its equilibrium position $d=L(F / W)$, where $L$ is the pendulum length, $F$ is the force produced. and W is the thruster's weight.

In order to increase our ability to detect any motion of the thruster, a simple lens (focal length = 4") was attached to the thruster, and the beam from a small laser was passed through the lens and onto an opposite wall. The displacement of the spot on the wall, D, is related to the thruster displacement $\mathrm{d}$, by the relation $\mathrm{D}=$ $(R / f) d$, where $R$ is the distance from the lens to the wall and $f$ is the focal length of the lens. In our case $R=9^{\prime} 4^{\prime \prime}$ and $f=4$ ", so the magnification factor $R / f=28$.

With this setup, we could estimate the upper limit for the thrust produced by the antenna. Occasianally there was spot displacement of perhaps a centimeter upon initial energizing of the thruster. It was not repeatable during a test session, and we attributed it to momentary stiffening of the power cable. The power supply was equipped with a switch that allowed us to apply power to the thruster in bursts. We tried to apply power at the natural frequency of the pendulum, in hope of seeing a greater displacement, but to no avail.

Thus we (generously) take the maximum observable spot displacement as $1 \mathrm{~cm}$. This translates to a pendulum displacement of $\left(10^{-2} \mathrm{~m}\right) / 28=3.6 \times 10^{-4} \mathrm{~m}$. The length of the pendulum was $88^{\prime \prime}=2.2 \mathrm{~m}$, so the ratio $\mathrm{d} / \mathrm{L}$ is $\left(3.6 \times 10^{-4} \mathrm{~m}\right) /(2.2 \mathrm{~m})=1.6 \times 10^{-4}$. The weight of the thruster is $1.8 \mathrm{~kg} \times 9.8 \mathrm{~ms}^{-2}=17.6$ newtons, so the upper limit for the thruster force is $17.6 \mathrm{~N} \times 1.6 \times 10^{-4}=2.8 \times 10^{-3} \mathrm{~N}=6.3 \times 10^{-4}$ lbf $=$ $1.0 \times 10^{-2}$ ozf. This is considerably less than the force expected by the authors: 0.03 to $0.3 \mathrm{~N}$ at 4 amps, 28 volt dc. (Ref. 5). But, in a sense, the amount of thrust produced by this device is irrelevant to spacecraft propulsion. That is the topic of the next section.

\section{Is There Hope for a Space Drive Within Classical Electrodynamics?}

We should recognize, and the earlier the better, that there is little hope for a propulsion scheme that appears as a space reaction drive to an external inertial observer and is based only on the exceedingly well established Maxwellian electrodynamics. The heart of the matter is that classical electrodynamics and mechanics requires conservation of momentum in flat spacetime. Without the existence of an ether-like medium that could sustain volume forces, the momentum of an isolated electrodynamic system (matter plus the EM fields it generates) is strictly conserved. And of course, Maxwell's equations are incompatible with that kind of ether. Thus a scheme based on classical concepts can be at most a photon rocket.

Since space reaction schemes based just on EM fields keep being proposed, it may be worthwhile to review the derivation of the EM momentum theorem in order to appreciate its compelling nature. Taking the macroscopic view of material properties, formal 
manipulation of Maxwell's equations leads to the divergence relation

$$
\nabla \cdot \overrightarrow{\mathrm{T}}=\rho \overrightarrow{\mathrm{E}}+\overrightarrow{\mathrm{J}} \times \overrightarrow{\mathrm{B}}+\frac{\partial}{\partial \mathrm{t}}(\overrightarrow{\mathrm{D}} \times \overrightarrow{\mathrm{B}})
$$

where

$$
\overrightarrow{\mathrm{T}} \equiv \overrightarrow{\mathrm{E}} \overrightarrow{\mathrm{D}}+\overrightarrow{\mathrm{H}} \overrightarrow{\mathrm{B}}-\frac{1}{2}(\overrightarrow{\mathrm{E}} \cdot \overrightarrow{\mathrm{D}}+\overrightarrow{\mathrm{H}} \cdot \overrightarrow{\mathrm{B}}) \overrightarrow{\mathrm{I}}
$$

is the Maxwell stress tensor (in dyadic notation). This form of $\overline{\mathrm{T}}$ neglects the various internal stresses in matter, due to magneto- and electro-striction and nonuniformity of polarizabilities; however, these terms do not contribute to the volume integral of $\nabla \cdot \vec{T}$.

Although the terms in the $\nabla \cdot \overrightarrow{\mathrm{T}}$ have the dimensions of force density and $\overrightarrow{F_{v}} \equiv \rho \vec{E}+\vec{J} \times \vec{B}$ is clearly the usual Lorentz force density on matter, nothing in the derivation requires that every term in $\nabla \cdot \overrightarrow{\mathrm{T}}$ be interpreted as a force density. Forcing such an interpretation on $\frac{\partial}{\partial t}(\vec{D} \times \vec{B})$ suggests the dubious notion of a force on EM fields or even space itself! It may be best to avoid such interpretations, with their ether-like notions, and to proceed with the integration leading to the momentum theorem, as per standard textbooks in electrodynamics (Ref. 6). The physical interpretation is then thrown back to well established concepts. Under the assumption that the Lorentz force is the only force on the matter in our electrodynamic system, the rate of change of its mechanical momentum is just

$\frac{\mathrm{d} \overrightarrow{\mathrm{p}}}{\mathrm{dt}}=\iiint_{\mathrm{v}} \overrightarrow{\mathrm{F}}_{\mathrm{v}} \mathrm{dv}$,

where the volume of integration $\mathrm{V}$ encloses the system. Hence in this notation.

$$
\frac{\mathrm{d}}{\mathrm{dt}}\left[\overrightarrow{\mathrm{p}}+\iiint_{\mathrm{v}}(\overrightarrow{\mathrm{D}} \times \overrightarrow{\mathrm{B}}) \mathrm{dv}\right]=\oiint_{\mathrm{A}} \overrightarrow{\mathrm{T}} \cdot \hat{\mathrm{n}} \mathrm{dA} \text {. }
$$

Letting the surface $A$ of $V$ expand in all directions, the above surface integral vanishes rigorously. To see this, observe that the nonradiative components of the fields decay faster than $1 / \mathrm{r}$ with distance, thus contributing terms to $\overrightarrow{\mathrm{T}} \cdot \hat{\mathbf{n}}$ that decay faster than $1 / \mathrm{r}^{2}$. And if the surface is sufficiently far away, then any radiative fields turned on at a finite time in the past have not yet reached the surface - all photons are caught within the surface and taken into account. The result is the well- known, and relativistically valid, momentum theorem: $\overrightarrow{\mathrm{p}}+\iiint_{\infty}(\overrightarrow{\mathrm{D}} \times \overrightarrow{\mathrm{B}}) \mathrm{dv}=$ const.

The above volume integral is easily interpreted as the total momentum in the EM field and for free space, the integrand reduces to the familiar $(\overrightarrow{\mathrm{E}} \times \overrightarrow{\mathrm{H}}) / \mathrm{c}^{2}$.

This momentum conservation law clearly shows that from the point of view of classical mechanics, the validity of Maxwell's equations in flat spacetime limits the space drive to at best a photon rocket. One might try to argue such things as what is the EM momentum in matter etc., but this is at the level of 'tweaking' and not a solution to practical interstellar transport. This severe bottom line strongly suggests that for practical, globally fast mass/energy transport, one must work around the classical limitations of momentum conservation by digging into the deeper layers of spacetime structure itself - the so called "spacetime engineering". Quite likely, that will bring one to deal with the vacuum zeropoint fluctuations, the so-called virtual particle pairs and possibly other elements of the support of spacetime.

\section{Summary and conclusions}

No reproducible evidence was ever seen for any thrust when current pulses of $1200 \mathrm{~A}$ peak and $20 \mu$ s risetime were applied to the antenna at a rate of 30 per second. The result was still null, even when bursts of these pulses were applied in synch with the period of the pendulum-like suspension of the antenna. Our experiment could detect a steady force as low as $3 \mathrm{mN}$.

The simplicity and import of the electromagnetic momentum theorem underscore the hopelessness of any space reaction scheme strictly within classical electrodynamics. This severe bottom line strongly suggests that for practical, globally fast mass/energy transport, one must work around the classical limitations of momentum conservation by digging into the deeper layers of spacetime structure itself-the so called "spacetime engineering". Quite likely, that will bring one to deal with the vacuum zero-point fluctuations, the so-called virtual particle pairs and possibly other elements of the support of spacetime. 


\section{References}

1. R. L. Schlicher, et al., United States Patent 5,142,861, Nonlinear Electromagnetic Propulsion System and Method. Sept. 1, 1992

2. R. L. Schlicher, et al., Nonlinear Electromagnetic Propulsion System and Method, $19^{\text {th }}$ Power Modulation Symposium, IEEE, New York, 1990. pp. 139-145.

3. R. L. Schlicher, A. W. Biggs, W. J. Tedeschi, Mechanical Propulsion from Unsymmetrical Magnetic Induction Fields, $31^{\text {si }}$ AIAA/ASME/SAE/ASEE Joint Propulsion Conference and Exhibit, AIAA 95-2643. July, 1995.
4. R. L. Schlicher, A. W. Biggs, W. J. Tedeschi, "Antenna Geometry" Mechanical Propulsion from Unsymmetrical Magnetic Induction Fields, $31^{\mathrm{st}}$ AIAA/ASME/SAE/ASEE Joint Propulsion Conference and Exhibit. July, 1995.

5. R. L. Schlicher, A. W. Biggs, W. J. Tedeschi, "Theoretical Analysis", Mechanical Propulsion from Unsymmetrical Magnetic Induction Fields, $31^{\text {st }}$ AIAA/ASME/SAE/ASEE Joint Propulsion Conference and Exhibit, July, 1995.

6. W.K.H. Panofsky and M. Phillips, Classical Electricity and Magnetism, $2^{\text {nd }}$ Edition, AddisonWesley Publishing Co., 1962. pp. 181-184. 


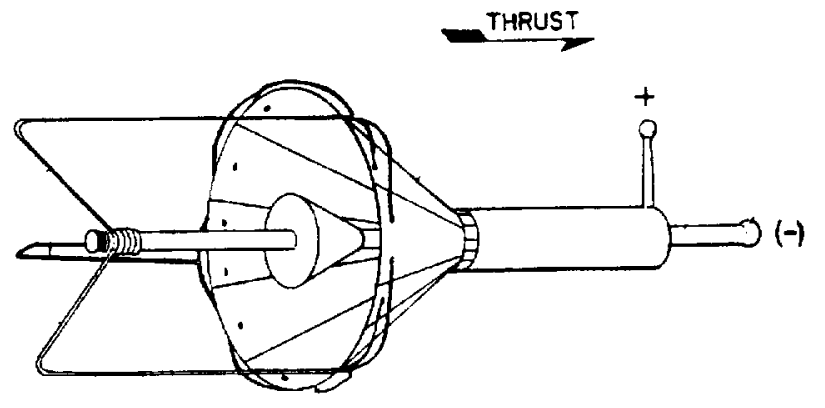

Figure 1. A typical NEMPS antenna, illustrated by R.L. Schlicher in his patent 5,142,861.



Figure 2. Our rendition of a NEMPS antenna, suspended from the ceiling. The coaxial Cable for current feed is visible on the right. Note also the lens for detection of motion by means of a laser beam. 


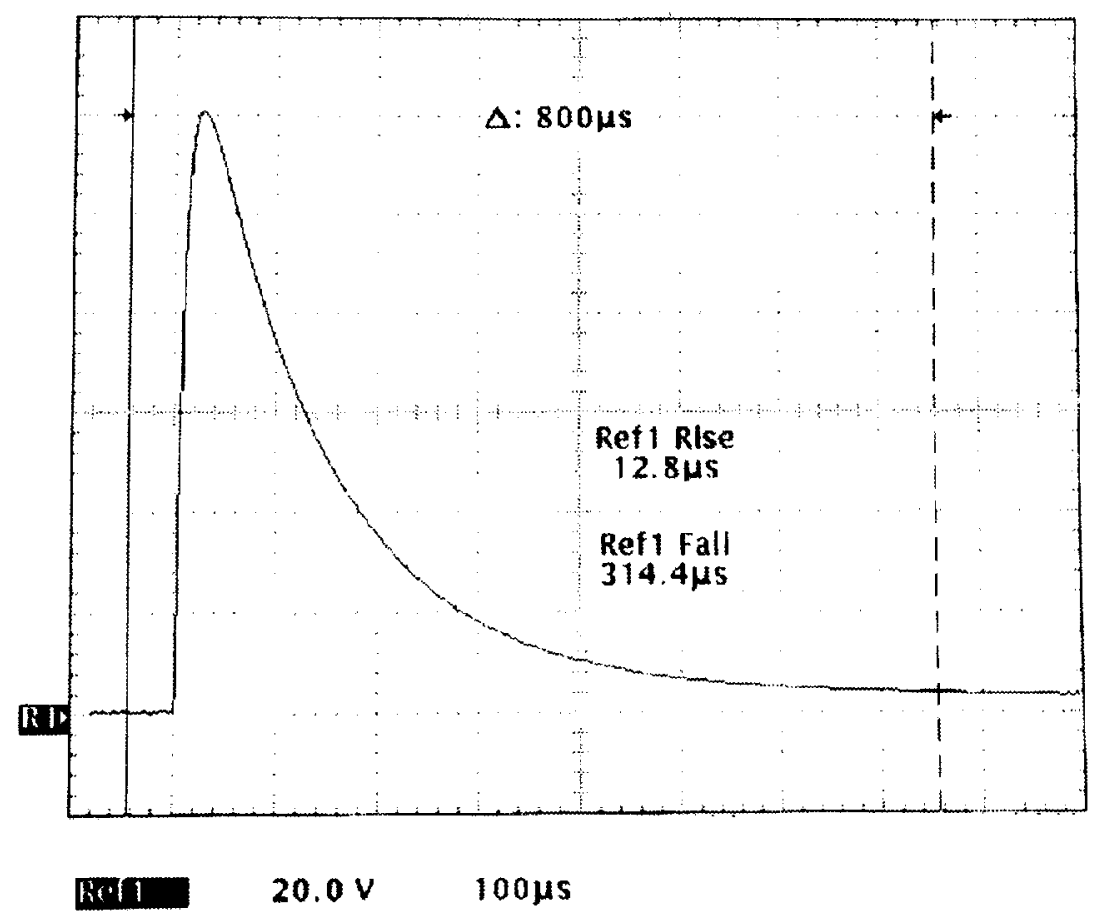

Figure 3. Typical pulse shape at maximum capability: 2400 A peak into a $0.05 \Omega$ resistive load. When driving the thruster antenna through our coaxial cable, the pulse height was reduced to about $1200 \mathrm{~A}$, at rise and fall times of about $20 \mu \mathrm{s}$ and $400 \mu \mathrm{s}$, respectively.

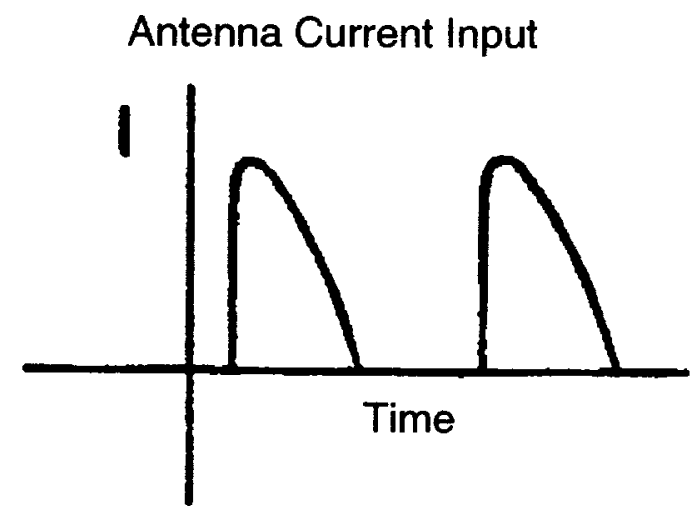

Figure 4. Current pulse shape sketch shown in the Schlicher patent. No scales are given. 


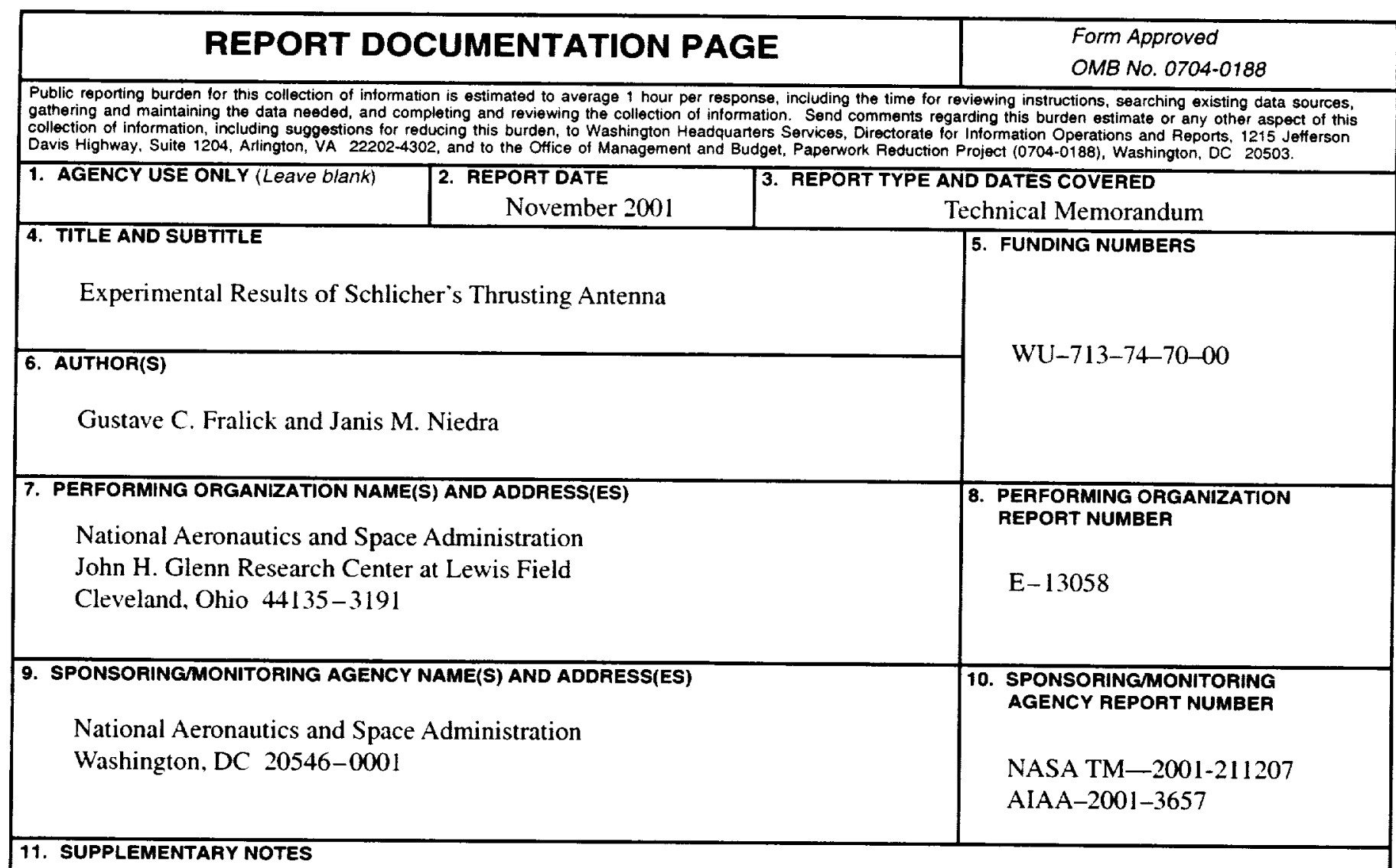

Prepared for the 37th Joint Propulsion Conference and Exhibit cosponsored by the AIAA, SAE, AIChE, and ASME, Salt Lake City, Utah. July 8-11, 2001. Gustave C. Fralick. NASA Glenn Research Center, and Janis M. Niedra, QSS Group, Inc., 2000 Aerospace Parkway, Brook Park, Ohio 44142. Responsible person. Gustave C. Fralick, organization code $5510,216-433-3645$.

\begin{tabular}{|l|l|}
\hline 12a. DISTRIBUTION/AVAILABILITY STATEMENT & 12b. DISTRIBUTION CODE
\end{tabular}

Unclassified - Unlimited

Subject Category: 07

Distribution: Nonstandard

Available electronically at http://gltrs.grc.nasagov/GLTRS

This publication is available from the NASA Center for AeroSpace Information. 301-621-0390.

13. ABSTRACT (Maximum 200 words)

Experiments were conducted to test the claims by Rex L. Schlicher, et al., (Patent 5,142,861) that a certain antenna geometry produces thrust greatly exceeding radiation reaction, when driven by repetitive, fast rise, and relatively slower decay current pulses. In order to test this hypothesis, the antenna was suspended by strings as a $3 \mathrm{~m}$ pendulum. Current pulses were fed to the antenna along the suspension path by a very flexible coaxial line constructed from loudspeaker cable and copper braid sheath. When driving the antenna via this cabling, our pulser was capable of sustaining $1200 \mathrm{~A}$ pulses at a rate of 30 per second up to a minute. In this way, bursts of pulses could be delivered in synch with the pendulum period in order to build up any motion. However, when using a laser beam passing through a lens attached to the antenna to amplify linear displacement by a factor of at least 25 , no correlated motion of the beam spot could be detected on a distant wall. We conclude, in agreement with the momentum theorem of classical electromagnetic theory, that any thrust produced is far below practically useful levels. Hence, within classical electrodynamics, there is little hope of detecting any low level motion that cannot be explained by interactions with surrounding structural steel and the Earth's magnetic field.

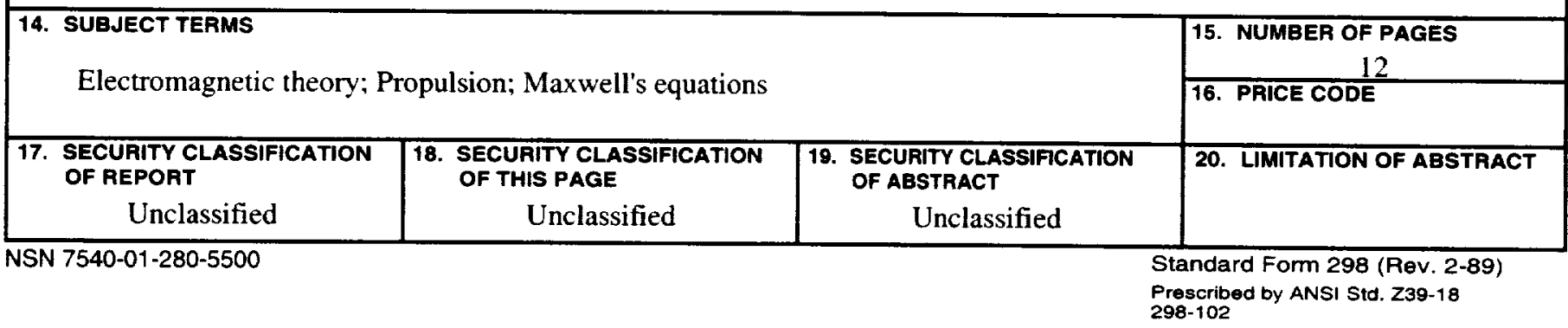





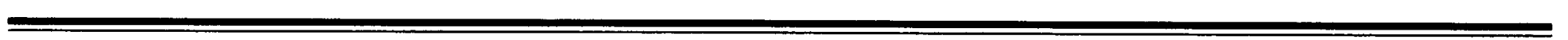

